\title{
Technology Transfer: A Previously Unsolved Problem
}

\author{
Robert A. Frosch
}

In Moliere's play Le Bourgeois Gentilhomme, Monsieur Jourdain is astonished to learn that he has been speaking prose all his life. Technology transfer has become all the rage recently, and some who have been practicing it for many years without having a name for what they were doing have been astonished to learn that what they were doing had a name. Part of the confusion has arisen from uncertainty about the meaning of "technology," a confusion between things that are embodiments of technology, and technology itself defined as "knowing how to do something." The technology is in the knowledge; the objects, processes, papers, and patents are only particular embodiments or abstracts of the knowledge.

Thus, giving, selling, or licensing technology to someone in the form of an embodiment or patent is unlikely to "transfer" the technology so that the recipient can really practice it and extend it. Such a "transfer" will work only if the recipient is skilled enough in the subject to understand the underlying knowledge and to use it without considerable further work just by examining the embodiment or the written material. This happens, but in my experience it is uncommon.

Thus technology transfer involves learning and dialogue. It is an activity engaged in by consenting adults: both parties must be involved participants. Technology transfer "at arms length" is generally impossible.
How does it occur? Most activities referred to today as "technology transfer" bear the same relationship to the real thing as a dating service does to a marriage: the service may make initial connections between people, but the people themselves must then decide what to do next. True technology transfer is problem solving. Sometimes the customer for technology seeks a technological solution to a problem which may be found in a research and development environment (technology pull).

\section{Technology transfer involves learning and dialogue.}

Sometimes a technology emerges from a research and development perspective because of expectations that it will be useful, or it results from (sometimes by chance or as a byproduct) a search for something else. Such a technology may seek a use either by finding an existing problem it can

Materials Matters is a forum for expressing personal points of view on issues of interest to the materials community. solve or by discovering a new problem it can solve (technology push).

In either case, it is unlikely that the technology, just as it exists, can be applied to the defined problem. It is much more likely that the technology will require further work and adjustments to be applied to a particular case, or redefinition or adjustment of the problem to match possible technological solutions. In most cases both the technology and the problem need to be worked on together in an intense dialogue. In this sense, "the customer is always wrong" and the "technologist is always wrong."

When most customers for technology state a problem, they are likely to do so in terms of their own perception of a possible solution, rather than being able to state the underlying problem, which might be redefined in terms of an available solution. For example, a request for a "stronger material" may be unsatisfiable because the request really is, or should be, for a stronger design, and a material to make it with. Putting a new material into an existing design is unlikely to be a good technological solution; most new materials require new design criteria to be really useful. When a technologist puts forward a solution to a problem, it is frequently a search for a use for the wonderful discovery just made in the laboratory. Many, perhaps most, technology transfer attempts that fail do so because this initial mismatch and disagreement is not followed by intense dialogue over the real nature of the problem and of the technological possibilities.

For these reasons, it is generally necessary for the technologist and the customer for technology to work closely together in defining and carrying out the "technology transfer." The lending or transfer of people, either from the technology organization to the customer (if learning the problem is key), or from the customer for technology to the technology creating organization (if learning and adjusting the technology is key) are frequently important to this process. Technology transfer is really an extension of research and development.

Robert A. Frosch is vice president of the General Motors Research and Environmental Staff.

The MRS Bulletin invites readers to send letters to the editor. Letters must be signed and should include affiliation, address, and phone number. Short, typed letters are preferred. Mail or fax letters to:

Editor, MRS Bulletin, Materials Research Society, 9800 McKnight Road, Pittsburgh, PA 15237, FAX: (412) 367-4373 


\section{AutoProbe" \\ Will Change The Way You Think About Atomic Force Microscopes}

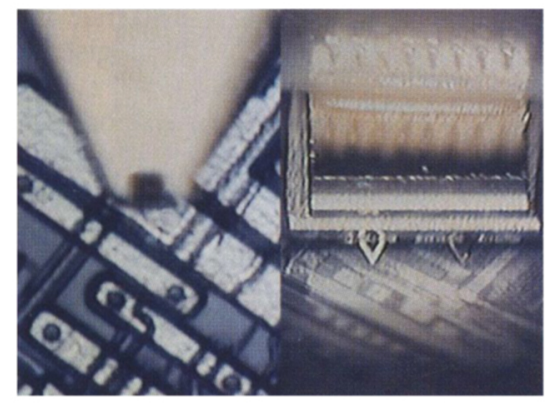

The highest-power, motorized-zoom, colorvideo, optical microscope system provides onaxis and oblique viewing with a field-of-view from $6 \mathrm{~mm}$ to $80 \mathrm{um}$.

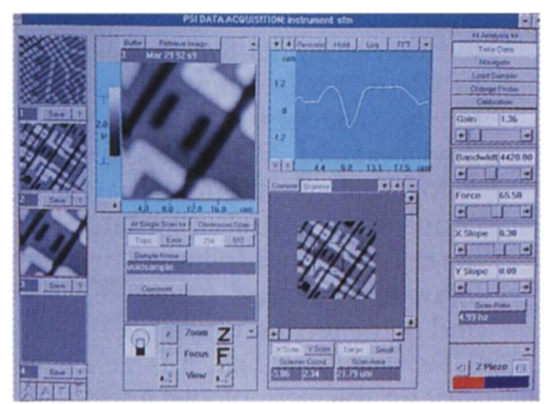

AutoProbe is easy to use through its intuitive, graphical user interface under Windows multitasking environment.

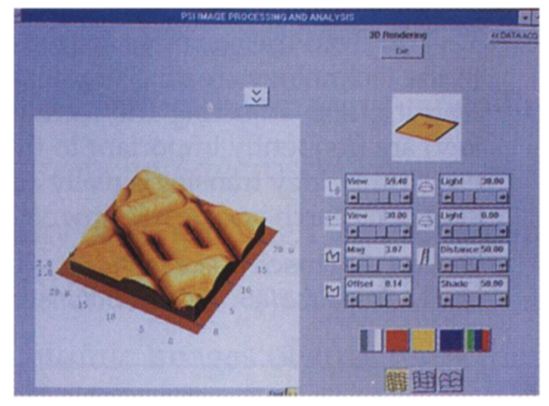

AutoProbe includes powerful data analysis and presentation software.

\author{
Simple and automated \\ operation. Unparalleled \\ performance. Advanced \\ data management. All \\ combined in a single \\ productivity tool. Not the \\ way you would normally \\ think about atomic force \\ microscopes. Until now.
}

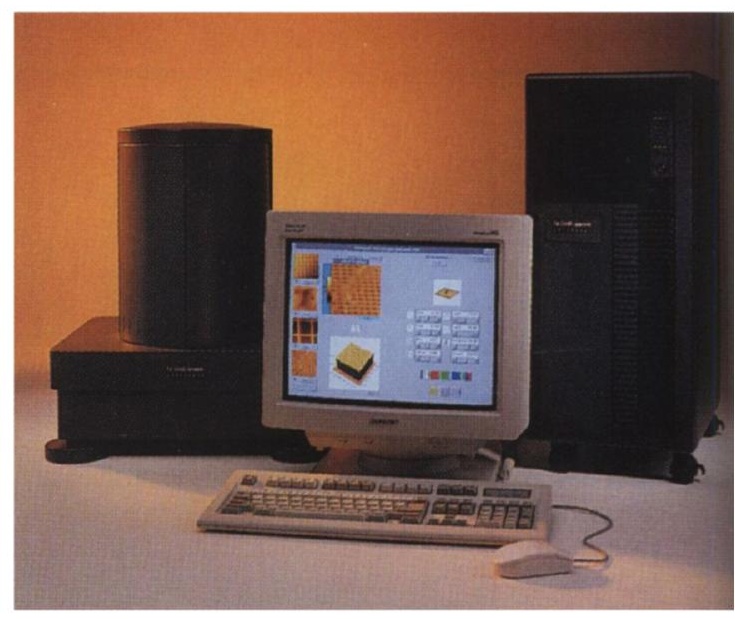

Operation is simple and automatic. AutoProbe's intuitive, graphical user interface runs in a Windows ${ }^{\text {TM }} 3.1$ multitasking environment. Advanced automation lets you load a new sample, insert a probe, and start producing high-quality images in less than a minute.

Performance is unmatched. AutoProbe's DSP-based control electronics are the most powerful available. The exclusive ScanMaster ${ }^{\mathrm{m}}$ closed-loop control system has position-sensitive detectors with $10 \AA$ resolution and $100 \mu \mathrm{m}$ scan range. AutoProbe delivers the industry's most accurate images - free from piezoelectric scanner non-linearity and hysteresis. DualView $^{\text {Th }}$ optics, another AutoProbe exclusive, provides seamless integration of optical and scanning probe microscopy.

Data management is flexible. Windows 3.1 with Paradox ${ }^{\mathrm{TM}}$ database software is the perfect platform for delivering fully integrated data analysis, presentation and handling capability. You can even compare and present data from multiple scans without additional off-line data processing.

For detailed information on AutoProbe, call 1-800-SPM-1602 today. We'll rush literature to you that will give you a whole new way to think about atomic force microscopes.

\section{¿Is Park Scientific Instruments ${ }^{\mathrm{Tm}}$}

1171 Borregas Avenue, Sunnyvale, CA 94089 Tel: 800-SPM-1602 Fax: 408-747-1601

In Japan: Hakuto Tel: 03-3225-8910 Fax: 03-3225-9011

In Europe: PSI S.A., Switzerland Tel: 41-22-300-4411 Fax: 41-22-300-4415 